\title{
Primary Central Nervous System Post-transplant Lymphoproliferative Disorder Diagnosed by Peripheral Facial Nerve Palsy
}

\author{
Aya Imafuku ${ }^{1}$, Kiho Tanaka ${ }^{1}$, Yuji Marui ${ }^{1}$, Yoshifumi Ubara ${ }^{1}$, Kenmei Takaichi ${ }^{1}$, \\ Shinji Tomikawa ${ }^{1}$, Yasunori Ota ${ }^{2,3}$, Takeshi Fujii ${ }^{2}$ and Yasuo Ishii ${ }^{1}$
}

\begin{abstract}
:
Although primary central nervous system post-transplant lymphoproliferative disorder (PCNS-PTLD) causes various symptoms depending on the tumor region, there has been no previous report of PCNS-PTLD in the cerebellopontine angle that was diagnosed due to peripheral facial nerve palsy. We herein report a case involving a 62-year-old man with PCNS-PTLD in the cerebellopontine angle who was diagnosed due to peripheral facial nerve palsy. The reduction of immunosuppressive therapy, whole-brain radiotherapy, intrathecal chemotherapy, and rituximab were effective in treating this patient. Physicians should therefore be mindful that PCNS-PTLD can cause peripheral facial nerve palsy in renal transplant recipients.
\end{abstract}

Key words: primary central nervous system post-transplant lymphoproliferative disorder, peripheral facial nerve palsy, cerebellopontine angle tumor

(Intern Med 57: 1899-1903, 2018)

(DOI: 10.2169/internalmedicine.9613-17)

\section{Introduction}

Although the most common cause of peripheral facial nerve palsy is Bell's palsy, a cerebellopontine angle tumor is also known to be a rare cause of this symptom (1). Primary central nervous system post-transplant lymphoproliferative disorder (PCNS-PTLD) causes various symptoms, including focal symptoms, headache, and seizure (2). To our knowledge, this is the first report of a rare case of PCNS-PTLD in the cerebellopontine angle that was diagnosed due to peripheral facial nerve palsy.

\section{Case Report}

A 62-year-old man was admitted to our hospital with left peripheral nerve palsy 4 years after he underwent living kidney transplantation. The cause of end-stage renal disease was diabetic nephropathy. Basiliximab, tacrolimus, mycophenolate mofetil (MMF), and prednisolone were adminis- tered as induction therapy. He had been treated with acyclovir against herpes zoster of the trigeminal nerve six months prior to admission. On admission, he was administered $3 \mathrm{mg}$ of tacrolimus daily, 1,500 $\mathrm{mg}$ of MMF daily, and $15 \mathrm{mg}$ of prednisolone daily. The target trough level for tacrolimus was $5 \mathrm{mg} / \mathrm{dL}$ as maintenance therapy. His height and body weight were $181 \mathrm{~cm}$ and $84 \mathrm{~kg}$, respectively. His blood pressure was $109 / 66 \mathrm{mmHg}$, pulse rate was 65 beats/ min, and temperature was $36.7^{\circ} \mathrm{C}$. A neurological examination revealed left peripheral palsy, shifted strain of the forehead, drooping of the eyebrow, incomplete lid closure, and drooping of the corner of the mouth; the patient also showed bilateral sensorineural hearing loss. There were no other cranial nerve manifestations or skin blisters.

Laboratory tests revealed a leukocyte count of $6.8 \times 10^{3} / \mathrm{L}$, urea nitrogen level of $36 \mathrm{mg} / \mathrm{dL}$, creatinine level of 1.16 $\mathrm{mg} / \mathrm{dL}$, C-reactive protein level of $0.0 \mathrm{mg} / \mathrm{dL}$, lactate dehydrogenase level of $189 \mathrm{U} / \mathrm{L}$, and soluble interleukin-2 receptor level of $387 \mathrm{U} / \mathrm{mL}$. Non-enhanced brain magnetic resonance imaging (MRI) showed multiple lesions in the pons,

${ }^{1}$ Department of Nephrology Center, Toranomon Hospital, Japan, ${ }^{2}$ Department of Pathology, Toranomon Hospital, Japan and ${ }^{3}$ Department of Pathology, The University of Tokyo, Japan

Received: June 3, 2017; Accepted: October 1, 2017; Advance Publication by J-STAGE: February 9, 2018

Correspondence to Dr. Aya Imafuku, aya_im1119@yahoo.co.jp 

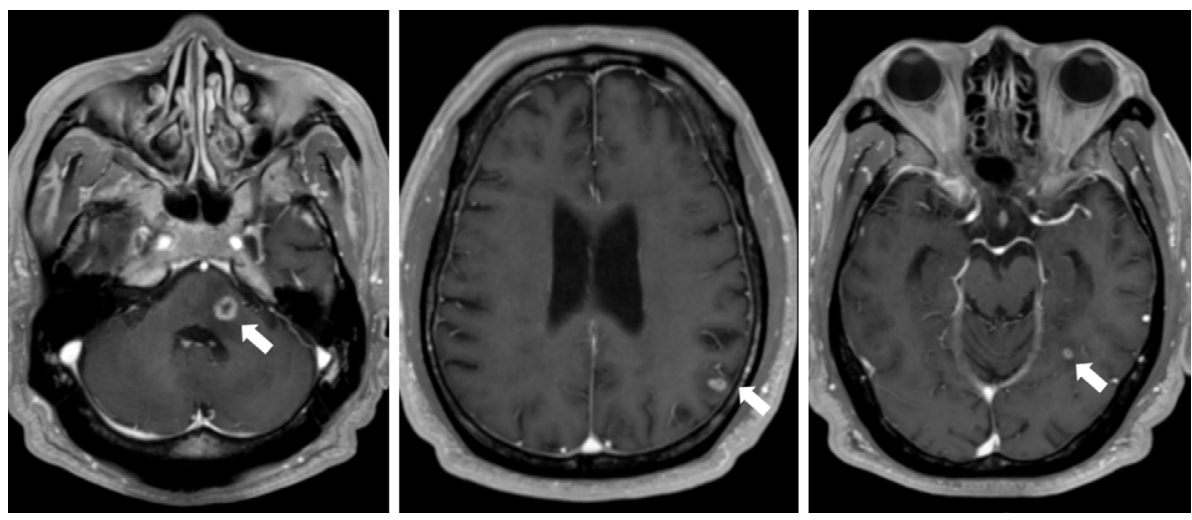

Figure 1. Gadolinium-enhanced brain magnetic resonance imaging. Gadolinium-enhanced brain MRI showed multiple ring-enhanced lesions in the left pons, left cerebellar peduncle, left temporal lobe, and parietal lobe.
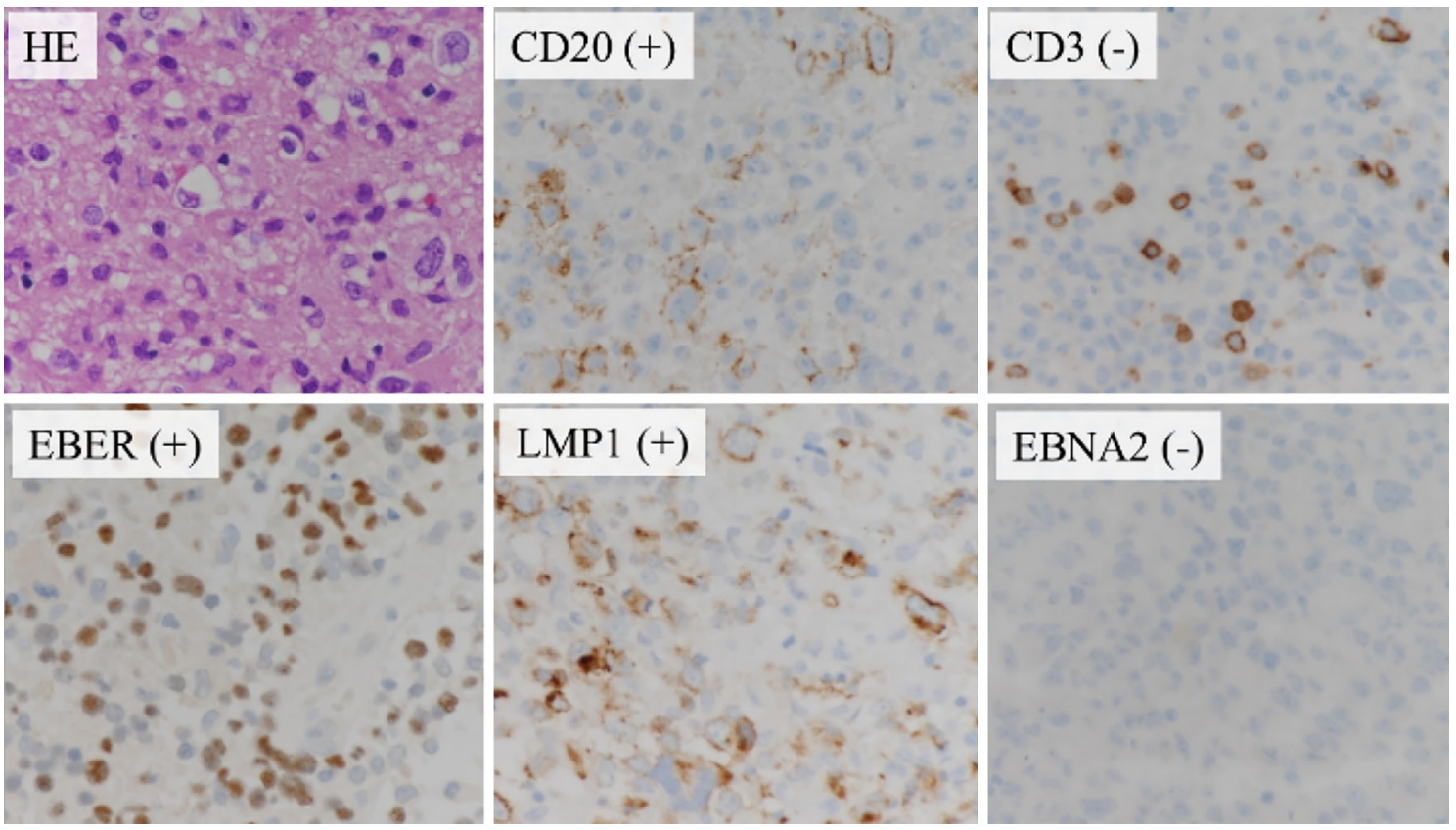

Figure 2. Pathology of brain tumor biopsy. Immunohistochemical analysis showed positive findings for CD20, EBER, LMP-1, and negative findings for CD3 and EBNA2.

temporal lobe, and parietal lobe on the left side. Cerebrospinal fluid (CSF) testing revealed normal cell counts, with a mild elevation of opening pressure and protein level.

Although acyclovir was initiated with suspicion of Bell's palsy, his symptom did not improve. Gadolinium-enhanced brain MRI showed multiple ring-enhanced lesions in the left pons, left cerebellar peduncle, left temporal lobe, and parietal lobe, which suggested the presence of metastatic tumors (Fig. 1). However, despite performing gastroscopy, colonoscopy, and positron emission tomography-computed tomography (PET-CT), there was no evidence of a primary lesion. On day 9, a serum quantitative polymerase chain reaction (PCR) analysis for Epstein Barr Virus (EBV)-DNA showed elevated results (400 copies/mL). EBV anti-Viral capsid antigen (VCA) IgG antibody was both positive in the donor and the recipient. Anti-human immunodeficiency virus
(HIV) antibody was negative. Therefore, we reduced the MMF dosage from $1,500 \mathrm{mg}$ to $500 \mathrm{mg}$ dairy and performed a brain tumor biopsy with suspicion of PTLD on day 14.

A pathological examination revealed monomorphic PTLD with primary diffuse large B-cell lymphoma of the central nervous system. An immunohistochemical analysis showed positive findings for CD20, CD30, B-cell lymphoma 2 (BCL 2), EBV encoded early RNAs (EBER), and Latent membrane protein 1 (LMP-1), B cell Oct binding protein 1 (BOB1), and octamer-binding transcription factor2 (OCT2) and negative findings for CD10, CD3, CD15, and Epstein Barr nuclear antigen 2 (EBNA2) (Fig. 2).

We performed magnetic resonance (MR) cisternography and CSF testing again for further investigation. MR cisternography revealed the presence of a cerebellopontine angle 
tumor in the right facial nerve, which was suggested to be the cause of the left peripheral facial nerve palsy (Fig. 3). The EBV-DNA level in the CSF was elevated at 4,300 copies/mL, but flow cytometry did not reveal any monoclonal cells. After the diagnosis of PCNS-PTLD, MMF was discontinued and substituted with everolimus (1.5 mg daily), and the tacrolimus dose was reduced to $2 \mathrm{mg}$ daily. The patient was treated with rituximab $\left(375 \mathrm{mg} / \mathrm{m}^{2}\right.$, once a week, 4 times in total), intrathecal methotrexate $15 \mathrm{mg}$ and cytarabine $40 \mathrm{mg}$, and subsequent whole-brain radiotherapy (40 Gy/20 Fr) (Fig. 4). His left facial nerve palsy partially recovered. A whole-blood EBV-DNA analysis showed negative findings at one month after treatment, and gadoliniumenhanced brain MRI showed resolution of multiple lesions

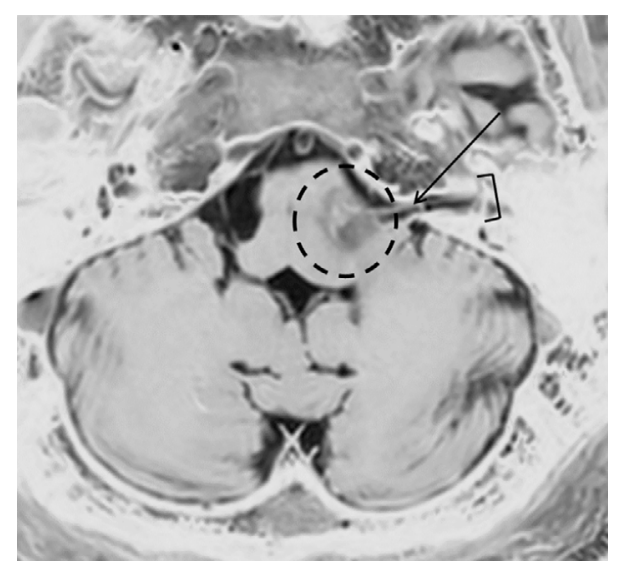

Figure 3. Magnetic resonance cisternography. MR cisternography revealed the presence of a cerebellopontine angle tumor in right facial nerve. Circle: Cerebellopontine angle tumor, Arrow: left facial nerve, Bracket: internal auditory meatus at five months after treatment. The patient is currently doing well without any relapse or deterioration of the kidney function at 18 months after treatment.

\section{Discussion}

We herein report the first case of PCNS-PTLD in the cerebellopontine angle that was diagnosed due to peripheral facial nerve palsy. A reduction of immunosuppressive therapy and the application of combination therapy of wholebrain radiotherapy, intrathecal methotrexate and cytarabine, and rituximab was effective in the present case.

Potential causes of peripheral facial palsy include infection, trauma, a tumor, autoimmune disease, or an idiopathic cause (1). Bell's palsy, which is included as an idiopathic cause, is the most common cause of peripheral facial palsy and accounts for approximately $75 \%$ of cases $(1,3)$. For comparison, neoplasm-induced facial paralysis accounts for only $5-10 \%$ of all cases $(4,5)$, and cerebellopontine angle tumors, such as an acoustic neurinoma or facial schwannoma, are a rare cause of peripheral facial paralysis (4).

The incidence of PTLD in the transplantation population has been estimated to be less than $2 \%$, and isolated CNS involvement is uncommon (6-8). In kidney transplant recipients, the incidence of PTLD is $1.2 \%$, and that of CNS involvement is $13 \%$ (7). Approximately $60 \%$ of patients with PCNS-PTLD have multiple lesions, and infratentorial involvement is seen in $4-33 \%$ of patients $(2,9)$. Although PCNS-PTLD causes various symptoms, including focal symptoms (82\%), headache (34\%), and seizure (29\%), there has been no previous report of associated peripheral nerve palsy (2). In enhanced MRI, lesions were enhanced in $97 \%$ of cases, with homogenous, heterogeneous, and ring patterns in $41 \%, 56 \%$, and $29 \%$ of cases, respectively (2). Thus,

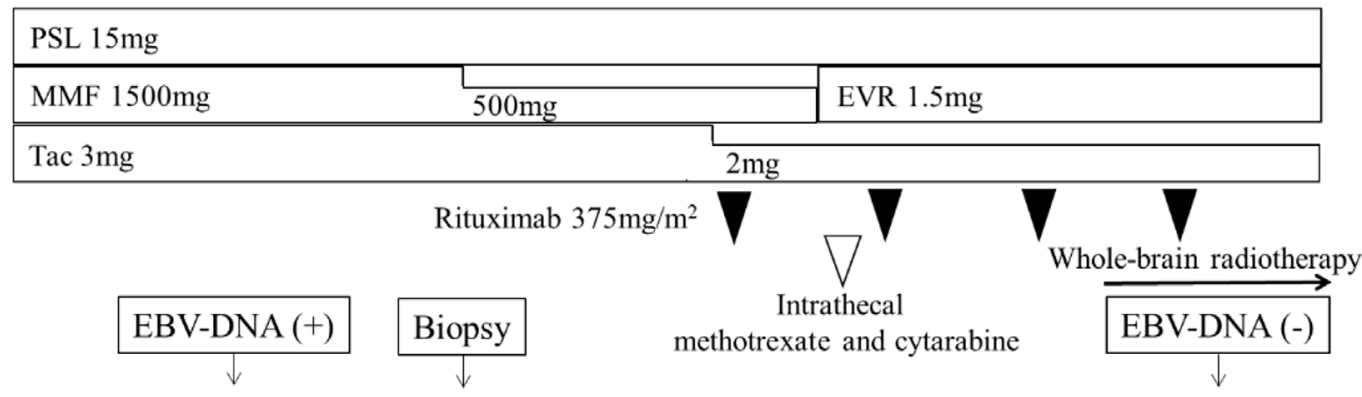

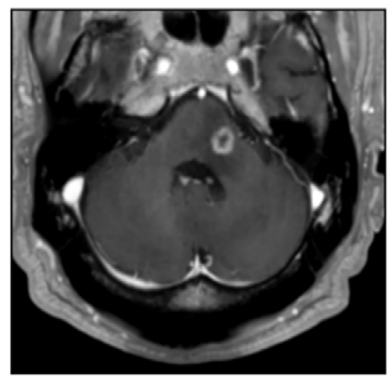

0

10
20

Figure 4. Clinical course.

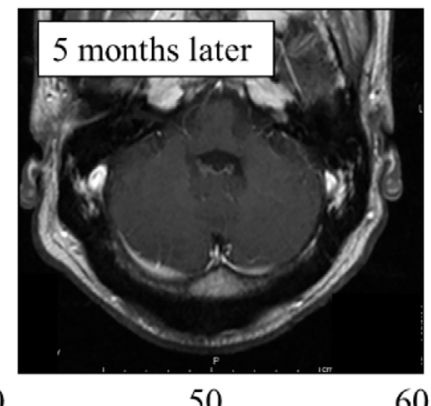

40

50

(day) 
physicians should note that PCNS-PTLD can be a cause of peripheral facial nerve palsy in renal transplant recipients and perform an appropriate brain imaging test.

In most cases, PTLD is associated with EBV infection of $B$ cells, either due to the reactivation of the EBV or primary EBV infection. The sensitivity for diagnosing PTLD based on an elevated EBV load in the blood is estimated to be over $90 \%$ (10), and monitoring of serum EBV-DNA is also useful for the evaluation of disease progress and the prediction of the prognosis (11). In most patients with CNSPTLD, EBV-DNA in blood is positive. Although information about EBV-DNA in the CSF is not available in many patients with CNS-PTLD, some patients show elevated levels of EBV-DNA in the $\operatorname{CSF}(8,12,13)$. However, some authors report that patients with CNS PTLD are negative for EBV-DNA in the blood $(14,15)$, or even show negativity for EBV-DNA in both the blood and CSF (16), suggesting that the diagnosis of PCNS-PTLD is difficult.

Although the optimum treatment for PCNS-PTLD has not been established, therapeutic options include reduction of immunosuppression, whole-brain radiotherapy, chemotherapy, and monoclonal antibody therapy. Since immunosuppression is recognized as a risk factor for PTLD, the reduction of immunosuppressive therapy is essential, taking into account the risk of rejection. Tumor regression has been induced in $50 \%$ of patients with systemic PTLD due to the reduction of immunosuppressive therapy alone, without any additional therapy (17). However, since achieving sufficient tumor regression by only reducing the dose of immunosuppressive therapy is rare in patients with CNS-PTLD, another type of treatment is therefore required (2). Everolimus, a mammalian target of rapamycin (mTOR) inhibitor, has been used for immunosuppression as well as for the treatment of cancer. Thus, mTOR inhibitors may have dual benefit as immunosuppressive drugs and as anti-tumor agents in patients with PTLD. Although the effect of mTOR inhibitor in patients with PTLD remains unclear, some authors have reported a low incidence of PTLD in transplant recipients receiving mTOR inhibitors (18). In addition, some reports have described the remission of PTLD after the induction of mTOR inhibitors (19-21). In our patients, we reduce the dose of MMF immediately when we suspect the possibility of PTLD. After the diagnosis of PTLD, we switch patients from MMF to everolimus and reduce the dose of tacrolimus. We adjust the dose of immunosuppressive drugs while carefully monitoring the kidney function.

Some authors have reported that whole-brain radiation therapy improved the survival (36 months vs. 7 months) in kidney transplant recipients with PCNS-PTLD (9). Furthermore, high-dose intravenous methotrexate, which is the standard treatment for primary CNS lymphoma, was applied to patients with CNS-PTLD $(22,23)$. However, the indication of high-dose intravenous methotrexate for CNS-PTLD in kidney transplant recipients seems limited due to the risk of nephrotoxicity. Although the benefits of intrathecal methotrexate for the survival in patients with primary CNS lymphoma have not been established, intrathecal methotrexate is often administered when the cytological results are positive (24). Some kidney transplant recipients with PCNS-PTLD have been treated with intrathecal methotrexate $(6,9)$. Although rituximab, an anti-CD20 monoclonal antibody, does not penetrate the blood-brain barrier effectively, some authors have reported that rituximab-based regimens improved the survival in patients with PCNSPTLD (2). However, the prognosis of patients with PCNSPTLD is known to be poor; indeed, Buell et al. (25) reported that the 3-year survival rate was $9.4 \%$, and Snanoudj et al. (9) reported that the median survival duration was 26 months.

In the current case, whole-brain radiotherapy, intrathecal chemotherapy, and rituximab were used. High-dose intravenous methotrexate was not used due to concerns over nephrotoxicity. The patient was doing well without any relapse or deterioration of the kidney function at 18 months after treatment. Thus, the combination of whole-brain radiotherapy, intrathecal chemotherapy, and rituximab seems to be a promising treatment option for PCNS-PTLD in kidney transplant recipients.

In conclusion, clinicians should therefore be aware that PCNS-PTLD can cause peripheral facial nerve palsy in renal transplant recipients.

\section{The authors state that they have no Conflict of Interest (COI).}

\section{References}

1. Finsterer J. Management of peripheral facial nerve palsy. Eur Arch Otorhinolaryngol 265: 743-752, 2008.

2. Cavaliere R, Petroni G, Lopes MB, Schiff D. Primary central nervous system post-transplantation lymphoproliferative disorder: an International Primary Central Nervous System Lymphoma Collaborative Group Report. Cancer 116: 863-870, 2010.

3. Peitersen E. Bell's palsy: the spontaneous course of 2,500 peripheral facial nerve palsies of different etiologies. Acta Otolaryngol Suppl 4-30, 2002.

4. Leonetti JP, Marzo SJ, Anderson DA, Sappington JM. Neoplastic causes of nonacute facial paralysis: a review of 221 cases. Ear Nose Throat J 95: 390-404, 2016.

5. Gupta S, Mends F, Hagiwara M, Fatterpekar G, Roehm PC. Imaging the facial nerve: a contemporary review. Radiol Res Pract 2013: 248039, 2013.

6. Lake W, Chang JE, Kennedy T, Morgan A, Salamat S, Baskaya MK. A case series of primary central nervous system posttransplantation lymphoproliferative disorder: imaging and clinical characteristics. Neurosurgery 72: 960-970, 2013.

7. Caillard S, Lelong C, Pessione F, Moulin B. Post-transplant lymphoproliferative disorders occurring after renal transplantation in adults: report of 230 cases from the French Registry. Am J Transplant 6: 2735-2742, 2006.

8. Castellano-Sanchez AA, Li S, Qian J, Lagoo A, Weir E, Brat DJ. Primary central nervous system posttransplant lymphoproliferative disorders. Am J Clin Pathol 121: 246-253, 2004.

9. Snanoudj R, Durrbach A, Leblond V, et al. Primary brain lymphomas after kidney transplantation: presentation and outcome. Transplantation 76: 930-937, 2003.

10. Tsai DE, Douglas L, Andreadis C, et al. EBV PCR in the diagno- 
sis and monitoring of posttransplant lymphoproliferative disorder: results of a two-arm prospective trial. Am J Transplant 8: 10161024,2008

11. van Esser JW, van der Holt B, Meijer E, et al. Epstein-Barr virus $(\mathrm{EBV})$ reactivation is a frequent event after allogeneic stem cell transplantation (SCT) and quantitatively predicts EBVlymphoproliferative disease following T-cell--depleted SCT. Blood 98: 972-978, 2001

12. Yaginuma T, Yamamoto H, Mitome J, et al. Successful treatment of monomorphic primary central nervous system posttransplantation lymphoproliferative disorder 5 years after kidney transplantation. Transpl Infect Dis 14: E102-E106, 2012.

13. Nozzoli C, Bartolozzi B, Guidi S, et al. Epstein-Barr virusassociated post-transplant lymphoproliferative disease with central nervous system involvement after unrelated allogeneic hematopoietic stem cell transplantation. Leuk Lymphoma 47: 167-169, 2006.

14. Boersma MN, van der Zanden A, Laverman GD, Sanders JS, de Vries PA. Epstein-Barr virus-positive post-transplant lymphoproliferative disorder of the central nervous system, after renal transplantation with a discrepancy in viral load between peripheral blood and cerebrospinal fluid. Transpl Int 25: e113-e116, 2012.

15. Terasawa T, Ohashi H, Tsushita K, et al. Failure to detect EpsteinBarr virus (EBV) DNA in plasma by real-time PCR in a case of EBV-associated posttransplantation lymphoproliferative disorder confined to the central nervous system. Int J Hematol 75: 416-420, 2002.

16. Hamadani M, Martin LK, Benson DM, Copelan EA, Devine SM, Hofmeister CC. Central nervous system post-transplant lymphoproliferative disorder despite negative serum and spinal fluid Epstein-Barr virus DNA PCR. Bone Marrow Transplant 39: 249251, 2007.

17. Paya CV, Fung JJ, Nalesnik MA, et al. Epstein-Barr virus-induced posttransplant lymphoproliferative disorders. ASTS/ASTP EBVPTLD Task Force and The Mayo Clinic Organized International
Consensus Development Meeting. Transplantation 68: 1517-1525, 1999.

18. Piselli P, Serraino D, Segoloni GP, et al. Risk of de novo cancers after transplantation: results from a cohort of 7217 kidney transplant recipients, Italy 1997-2009. Eur J Cancer 49: 336-344, 2013.

19. Cullis B, D’Souza R, McCullagh P, et al. Sirolimus-induced remission of posttransplantation lymphoproliferative disorder. Am J Kidney Dis 47: e67-e72, 2006.

20. Boratynska M, Smolska D. Inhibition of mTOR by sirolimus induces remission of post-transplant lymphoproliferative disorders. Transpl Int 21: 605-608, 2008.

21. Ferreira H, Bustorff M, Santos J, et al. Post-transplant lymphoproliferative disorder: a single-center experience. Transplant Proc 47: 981-984, 2015.

22. Hiraga S, Arita N, Ohnishi $\mathrm{T}$, et al. Rapid infusion of high-dose methotrexate resulting in enhanced penetration into cerebrospinal fluid and intensified tumor response in primary central nervous system lymphomas. J Neurosurg 91: 221-230, 1999.

23. Taj MM, Messahel B, Mycroft J, et al. Efficacy and tolerability of high-dose methotrexate in central nervous system positive or relapsed lymphoproliferative disease following liver transplant in children. Br J Haematol 140: 191-196, 2008.

24. Khan RB, Shi W, Thaler HT, DeAngelis LM, Abrey LE. Is intrathecal methotrexate necessary in the treatment of primary CNS lymphoma? J Neurooncol 58: 175-178, 2002.

25. Buell JF, Gross TG, Hanaway MJ, et al. Posttransplant lymphoproliferative disorder: significance of central nervous system involvement. Transplant Proc 37: 954-955, 2005.

The Internal Medicine is an Open Access article distributed under the Creative Commons Attribution-NonCommercial-NoDerivatives 4.0 International License. To view the details of this license, please visit (https://creativecommons.org/licenses/ by-nc-nd/4.0/).

(C) 2018 The Japanese Society of Internal Medicine Intern Med 57: 1899-1903, 2018 